\title{
A cross-sectional study of the nutritional status of community-dwelling people with idiopathic Parkinson's disease
}

\author{
Ahmed F Jaafar ${ }^{1 *}$, William K Gray², Bob Porter ${ }^{2}$, Elizabeth J Turnbull³ ${ }^{3}$ Richard W Walker ${ }^{2}$
}

\begin{abstract}
Background: Parkinson's disease (PD) patients have an increased risk of under-nutrition, but we are unaware of any population based prevalence studies of under-nutrition in PD. The main objective of this study was to identify the prevalence, and nature, of under-nutrition in a representative population of people with PD.

Methods: People diagnosed with idiopathic PD from within two PD prevalence study sites in North-East England were asked to participate in this study. Those who participated $(n=136)$ were assessed using a number of standard rating scales including Hoehn \& Yahr stage and Unified Parkinson's Disease Rating Scale (UPDRS). Body mass index (BMI), mid-arm circumference (MAC), triceps skin fold thickness (TSF) and grip strength were recorded together with social and demographic information.

Results: BMI < 20 identified over 15\% of the study group to have under-nutrition. The Malnutritional Universal Screening Tool (MUST) scoring system identified $23.5 \%$ of participants at medium or high risk of malnutrition. Low BMI, indicating under-nutrition, was associated with greater age and disease duration, lower MAC, TSF, mid-arm muscle circumference (MAMC), reduced grip strength and a report of unintentional weight loss. Problems increased with increasing age and disease duration and were greater in females.

Conclusions: Under-nutrition is a problem for around 15\% of community dwelling people with PD. All PD patients should be screened for under-nutrition; the MUST score is a useful early screening tool.
\end{abstract}

\section{Background}

Parkinson's disease (PD) is a progressive neurodegenerative disorder that has been shown to put individuals at increased risk of malnutrition and weight loss compared to age matched controls [1]. The nutritional problems associated with PD have been reviewed recently [2]. Previous studies have shown an increase in basal metabolic rate in PD patients, and this increase in energy expenditure has been implicated in the aetiology of weight loss in patients with PD [3-6]. Other possible causes of weight loss include, difficulty swallowing $[7,8]$, inability to prepare food and handle it appropriately [9], gastrointestinal symptoms of the disease itself and drug side effects, such as dyskinesia $[10,11]$.

\footnotetext{
* Correspondence: ahmed.jaafar@nuth.nhs.uk

'Department of Medicine, The Royal Victoria Infirmary, Newcastle upon Tyne, NE1 4LP, UK

Full list of author information is available at the end of the article
}

Using the mini nutritional assessment as a screening tool, a recent 3 year longitudinal study of 61 PD patients from a referral centre found malnutrition risk increased from $22.9 \%$ to $34.3 \%$. Greater disease duration was a significant predictor of risk from malnutrition [12]. The authors concluded that PD patients should be screened for nutritional status. Previous studies involving anthropometric measurement of PD patients have selected patients who were not representative of the wider PD community. Davies et al [5] studied 15 PD patients who had reported weight loss in the last 12 months and 15 controls. Although PD patients had significantly less body fat than controls, the authors noted the daily calorie intake of the PD patients to be significantly higher than the control subjects. We are unaware of any previous studies of the prevalence of nutritional problems in prevalent populations of people with PD.

The aim of this study was to assess the prevalence of under-nutrition in community-dwelling people with PD
C Biomed Central

C 2010 Jaafar et al; licensee BioMed Central Ltd. This is an Open Access article distributed under the terms of the Creative Commons Attribution License (http://creativecommons.org/licenses/by/2.0), which permits unrestricted use, distribution, and reproduction in any medium, provided the original work is properly cited. 
by use of standard anthropomorphic tests. Furthermore, we investigated whether levels of under-nutrition could be linked to demographic influences (age, sex, abode) and wider disease characteristics such as disease progression, disease duration, cognitive function, reported swallow problems and dyskinesia.

\section{Methods}

In previously published prevalence studies in North-East England we identified 161 eligible cases of PD within an urban study population of 108,597 in the North Tyneside area and 106 cases within a predominantly rural study population of 59,613 in North Northumberland [13.14]. A nutritional investigation was carried out as part of these studies. Background data relating to age, gender, disease duration, functional limitations, motor and cognitive skills, disability, anxiety, depression, and quality of life were available for those who were eligible, and agreed to participate. For the nutritional component of the study, a proportion of the study population either declined to take part or was otherwise unable to participate.

All participants were assessed at home by a member of the study team. In North Tyneside this was a physician with an interest in movement disorders (B.P.) [13] and in North Northumberland this was either a physician with an interest in movement disorders or a PD nurse specialist [14]. Diagnosis of PD was confirmed using the UK PD Brain bank criteria [15]. Demographic information (age, sex, abode, duration of disease), Hoehn and Yahr stage (degree of disability and functional limitation), [16] and Unified Parkinson's Disease Rating Scale (UPDRS) score [17] was recorded from patient responses to verbal questioning, examination and assessment according to standard protocols. The researchers had all received training in the use of the UPDRS. The UPDRS is designed to measure and monitor progression in all aspects of PD and focuses on four key areas: 1) mentation, behaviour and mood, 2) coping with activities of daily living, 3) motor function and 4) changes due to complications of therapy. Cognition was assessed by administering the Mini-Mental State Examination (MMSE) [18].

Collection of nutritional data involved measurement of weight using calibrated portable scales and height using a steel tape measure. Patients were weighed without shoes, but with indoor clothing; no attempt was made to subtract the weight of the clothing. Patients' height was measured with their shoes removed. BMI was calculated using the formula weight $(\mathrm{kg}) / \mathrm{height}^{2}(\mathrm{~m})$. A BMI of $<20$ was taken as an indicator of under-nutrition, and a BMI of $<18.5$ as severe under-nutrition $[19,20]$ Mid-arm circumference (MAC), triceps skin fold thickness (TSF), and mid-arm muscle circumference (MAMC) were recorded. MAC was measured in $\mathrm{cm}$ at the mid-point between the olecranon and acromion process of the right arm. TSF was measured in $\mathrm{mm}$ at the same point using Harpenden calipers; 3 measurements were taken and then the mean value used for analysis. MAMC was calculated in $\mathrm{cm}$ using the formula MAC- $(0.314 \times$ TSF) [21]. Grip strength of the right upper limb was measured in kilograms using a dynamometer; the greatest value from 3 attempts was used in the analysis.

Patient reported problems in swallowing food, liquids or tablets (yes or no); alcohol intake (units of alcohol per week), whether the patient smoked or had ever smoked (yes or no) and unintentional weight loss of $>$ $5 \%$ in the last 6 months (yes or no) were noted as these may contribute to under-nutrition. Other patient reported health problems were also noted. BMI and unintentional weight loss were used to calculate risk of malnutrition according to the Malnutrition Advisory Group's Mal-nutrition Universal Screening Tool (MUST) criteria [22].

This study was approved by the Joint Ethical Committee of Newcastle and North Tyneside and by the Northumberland Ethics Committee. Written informed consent was obtained from all subjects.

\section{Statistics}

The data were quantitative in nature and collected at a nominal, ordinal and interval/ratio level. They were analyzed using standard statistical software, SPSS-17 for windows (SPSS, Chicago, IL, USA). With the exception of age and UPDRS score, all predictor variables were found to be non-normally distributed (KolmogorovSmirnov test) and so did not meet parametric assumptions. No obvious outlier or influential cases were identified. A cut off of $\mathrm{p}<0.05$ (two-sided test) for statistical significance was used throughout. Mann-Whitney $\mathrm{U}$ test was used to establish differences in age, disease duration, MMSE score, UPDRS score and Hoehn and Yahr stage for people for whom nutritional data were collected and for those whom this data were not collected; in the case of gender a Chi-squared test was used. Correlation between predictor variables were assessed using Spearman's method for ordinal and inter$\mathrm{val} /$ ratio data, and using a point biserial method when one of the variables was dichotomous (gender, report of unintentional weight loss, dyskinesia present, BMI $<20$ ).

\section{Results}

Of the 161 prevalent cases of PD identified in North Tyneside, 135 (83.8\%) consented to be assessed. Of these, 10 patients were excluded due to having a level of cognitive impairment that rendered anthropomorphic testing impossible; a further 2 patients were excluded due to physical disability. Due to the limited time and 
resources nutritional data were not collected on patients towards the end of the study period, but this should not have introduced any bias into the results as patients were seen in an arbitrary order. Nutritional data was collected on 82 of the remaining 123 patients $(60.7 \%, 34$ males, and 48 females). Of the 106 prevalent cases of PD identified in North Northumberland, 75 (70.8\%) consented to be assessed, of whom 54 patients $(72.0,25$ males, and 29 females) underwent nutritional assessment. There were no significant differences in age, sex, disease duration, disease stage (Hoehn and Yahr), cognitive function (MMSE) or disease progression (UPDRS score) between those who had nutritional data completed and those who did not $(\mathrm{n}=53$ for North
Tyneside, $\mathrm{n}=21$ for North Northumberland), see Table 1. Therefore, we do not feel the fact that we could not assess all patients has biased our results in any significant way.

\section{Comparison of data for North Tyneside and North \\ Northumberland}

For those with nutritional data available, there was no significant difference in gender between those living in North Tyneside and those living in North Northumberland $\left(\chi^{2}(1)=0.310, p=0.600\right)$. Likewise, as shown in Table 1 age, disease duration, Hoehn and Yahr stage, BMI and grip strength were not significantly different between patients from North Northumberland and

Table 1 Comparison of PD patients who underwent all nutritional and anthropometric tests with those who did not

\begin{tabular}{|c|c|c|c|c|c|c|c|c|}
\hline & & Age & $\begin{array}{l}\text { Disease } \\
\text { duration }\end{array}$ & $\begin{array}{l}\text { Hoehn and } \\
\text { Yahr stage }\end{array}$ & MMSE score & UPDRS score & BMI & $\begin{array}{c}\text { Grip } \\
\text { strength (kg) }\end{array}$ \\
\hline \multicolumn{9}{|l|}{ North Tyneside } \\
\hline \multirow{4}{*}{$\begin{array}{l}\text { Data available }(\mathrm{n}=82 \text {, } \\
60.7 \%) \\
34 \text { males, } 48 \text { females }\end{array}$} & Mean & 74.59 & 5.61 & 2.49 & 26.80 & 30.96 & 24.88 & 18.72 \\
\hline & $\begin{array}{l}\text { Std } \\
\text { dev. }\end{array}$ & 8.837 & 5.060 & 1.048 & 4.915 & 14.993 & 4.825 & 9.257 \\
\hline & Range & $50-96$ & $1-28$ & $1-5$ & 0-30 & 4-89 & $16.2-40.3$ & $0-41$ \\
\hline & $\begin{array}{l}95 \% \\
\mathrm{Cl}\end{array}$ & $72.7-76.5$ & $4.5-6.7$ & $2.3-2.7$ & $25.7-27.9$ & $27.7-34.3$ & $23.8-25.9$ & $16.7-20.7$ \\
\hline \multirow{4}{*}{$\begin{array}{l}\text { No data available }(\mathrm{n}= \\
53,39.3 \%) \\
32 \text { males, } 21 \text { females }\end{array}$} & Mean & 75.83 & 5.79 & 2.60 & 23.76 & 33.59 & - & - \\
\hline & $\begin{array}{l}\text { Std } \\
\text { dev. }\end{array}$ & 7.967 & 3.857 & 1.050 & 8.941 & 16.368 & - & - \\
\hline & Range & 53-95 & $1-17$ & $1-5$ & $0-30$ & 11-79 & - & - \\
\hline & $\begin{array}{l}95 \% \\
\mathrm{Cl}\end{array}$ & 73.7-78.0 & $4.7-6.8$ & $2.3-2.9$ & $21.3-26.2$ & $29.0-38.2$ & - & - \\
\hline \multicolumn{9}{|l|}{ North Northumberland } \\
\hline \multirow{4}{*}{$\begin{array}{l}\text { Data available }(n=54, \\
72.0 \%) \\
25 \text { males, } 29 \text { females }\end{array}$} & Mean & 75.33 & 4.52 & 2.60 & 25.09 & 36.96 & 26.49 & 16.40 \\
\hline & $\begin{array}{l}\text { Std } \\
\text { dev. }\end{array}$ & 9.248 & 4.178 & 1.068 & 5.586 & 13.836 & 5.228 & 6.791 \\
\hline & Range & 53-93 & 0-17 & $1-5$ & 9-30 & 8-82 & $18.6-45.5$ & $5.0-40.0$ \\
\hline & $\begin{array}{l}95 \% \\
\mathrm{Cl}\end{array}$ & $72.8-77.9$ & $3.4-5.7$ & $2.2-3.0$ & $23.5-26.6$ & $33.2-40.7$ & $25.1-27.9$ & $14.5-18.3$ \\
\hline \multirow{4}{*}{$\begin{array}{l}\text { No data available } \\
(\mathrm{n}=21,28.0 \%) \\
13 \text { males, } 8 \text { females }\end{array}$} & Mean & 74.25 & 5.81 & 2.50 & 26.84 & 38.62 & - & - \\
\hline & $\begin{array}{l}\text { Std } \\
\text { dev. }\end{array}$ & 10.928 & 4.424 & 0.829 & 2.734 & 22.769 & - & - \\
\hline & Range & 47-89 & 0-15 & $1-4$ & $22-30$ & 10-88 & - & - \\
\hline & $\begin{array}{l}95 \% \\
\mathrm{Cl}\end{array}$ & $69.3-79.2$ & $3.8-7.8$ & $2.1-2.9$ & $25.5-28.2$ & $28.3-49.0$ & - & - \\
\hline $\begin{array}{l}\text { Difference between North } \\
\text { Tyneside and North } \\
\text { Northumberland* }\end{array}$ & & $\begin{array}{c}U=2036.5, z \\
=-0.790, p= \\
0.432\end{array}$ & $\begin{array}{c}U=1787.5, z \\
=-1.900, p= \\
0.057\end{array}$ & $\begin{array}{c}U=988.0, z \\
=-0.570, p= \\
0.572\end{array}$ & $\begin{array}{l}U=1739.5, z \\
=-1.980, p= \\
0.048\end{array}$ & $\begin{array}{c}U=1679.0, z \\
=-2.381, p= \\
0.017\end{array}$ & $\begin{array}{l}U=1845.0, z \\
=-1.641, p= \\
0.101\end{array}$ & $\begin{array}{c}U=1900.5, z \\
=-1.058, p= \\
0.292\end{array}$ \\
\hline
\end{tabular}

\footnotetext{
* For those with data available.
} 
North Tyneside. Mean MMSE score was 2 points lower and UPDRS score 6 points higher in North Northumberland. Given the broad similarity between the two groups, notably in terms of BMI and grip strength, data for North Tyneside and North Northumberland patients were combined for further analysis.

\section{Combined data set}

There was a negative correlation between BMI score and age $(\mathrm{r}=-0.273, \mathrm{p}=0.001)$, disease duration $(\mathrm{r}=$ -0.234, $\mathrm{p}=0.006$ ) and a report of unintentional weight loss by the patient $(r=-0.378, p<0.001)$. There was a positive correlation with grip strength $(\mathrm{r}=0.210, \mathrm{p}=$ $0.013)$ MAC $(\mathrm{r}=0.664, \mathrm{p}<0.001)$ TSF $(\mathrm{r}=0.349, \mathrm{p}<$ $0.001)$ and MAMC $(r=0.534, p<0.001)$. BMI score was not associated with smoking, alcohol consumption, dysphagia, dyskinesia, living in institutional care, UPDRS score or MMSE score.

Those who did have under-nutrition (BMI $<20, \mathrm{n}=$ $21)$ were compared to those who did not $(\mathrm{n}=115)$; results are given in Table 2; for anthropomorphic tests, results are split into males and females. Seven participants (5.15\%) had a BMI below 18.5, indicating severe under-nutrition, of whom 5 were female. Using the MUST scoring system 104 patients (56 females) were at low risk of malnutrition, 21 patients ( 11 females) were at medium risk of malnutrition and 11 (10 female) patients were at high risk of malnutrition. The odds of a female being at high risk of malnutrition compared to a male are 11.5 (95\% CI 1.43 to 93.0).

Table 3 compares our anthropomorphic data with data from two studies of the general elderly. One study is set in an industrial area of South Wales [23] in the early $1970 \mathrm{~s}$ and the other is from Edinburgh [24] in the mid-1990 s.

\section{Discussion}

This is the first published study, as far as we are aware, of nutritional problems in prevalent PD populations. Whilst the study is limited by a lack of data collection in a proportion of individuals it is reassuring that the disease characteristics of those in whom data was collected is not significantly different from those in whom data was not collected, as evident from the overlap of 95\% confidence intervals in all cases.

Although there is some debate as to the ideal BMI range for older people, a cut-off of $<20$ for undernutrition is widely recognised by most dieticians [25]. Moreover, a BMI of $<20$ has been suggested as a significant predictor of short-term mortality in older adults (mean age 73.5 years) [19]. Using BMI as a marker, under-nutrition was a problem in over $15 \%$ of community-dwelling people with PD. This compares with a report of only $3.6 \%$ under-nutrition $(\mathrm{BMI}<20)$ in an Italian study of nutrition in 3356 randomly selected elderly people (age range 65-84) [26]. Using the MUST criteria $23.5 \%$ of our cohort was at medium or high risk of malnutrition ( $8.1 \%$ at high risk). Considering females only these figures rise to $27.3 \%$ at medium to high risk, with $13.0 \%$ at high risk. Use of both BMI and a report of unintentional weight loss in the scoring system means that patients who still have an acceptable BMI, but are likely to become undernourished in the future if they continue to lose weight, are identified earlier than if BMI alone is used as a screening tool. This is likely to be particularly important in PD patients where weight loss may be due complex disease related factors, and therefore easier to control through dietary intervention if recognised at an early stage. Interestingly, unintentional weight loss was a stronger predictor of under-nutrition than patient reported swallowing problems.

We have not attempted to categorise patients according to degrees of malnourishment based on our anthropometric findings, since this would require the use of reference data from comparable normal populations. Data from 3 industrial areas of South Wales in the early 1970 s, although extensive, is likely to be out of date due to improving standards of health amongst the general elderly population over the last 30 years [23]. The average BMI of both males and females in our cohort was lower than that seen more recently in a study of elderly people in Edinburgh [24]. MAC, TSF and MAMC averages across both genders were generally higher than those recorded in South Wales, but lower than those recorded in Edinburgh. Although it is difficult to draw firm conclusions from these observations it may be that the Edinburgh cohort, being more recent, are a better match for our cohort and that our cohort are undernourished when compared to the general elderly population of a similar age.

Greater age and longer disease duration were significantly associated with under-nutrition in our cohort. Surprisingly, UPDRS score and Hoehn and Yahr stage, both markers of disease progression, were not significantly associated with under-nutrition. The reason for this is not clear, although comparison with previous research suggests there may be a link. The cohort of Davies et al [5] were of later Hoehn and Yahr stage than our cohort and had lower BMI and TSF and higher MAMC, indicating that late stage PD patients may have a greater degree of under-nutrition, with fat, rather than muscle, loss accounting for the change. Markus et al [27] studied 95 PD patients from a neurological unit. Despite the fact that the mean age of the cohort was only 61.7 years, the average Hoehn and Yahr stage was 3.0 and the average disease duration was 9.1 years, indicating the patients to be amongst the most severely 
Table 2 Comparison of those with BMI below 20 with those with BMI above 20

\begin{tabular}{|c|c|c|c|c|c|}
\hline & \multicolumn{2}{|c|}{$\begin{array}{c}\text { BMI }<20 \\
\text { (n = 21, 15.4\%) }\end{array}$} & \multicolumn{2}{|c|}{$\begin{array}{c}\text { BMI } \geq 20 \\
(n=115,84.6 \%)\end{array}$} & \multirow[b]{2}{*}{ Significance } \\
\hline & Median & Range & Median & Range & \\
\hline All participants & \multicolumn{2}{|c|}{6 male, 15 female } & \multicolumn{2}{|c|}{53 male, 62 female } & $\chi^{2}(1)=2.218, p=0.137$ \\
\hline Age & 81 & $70-92$ & 75 & $50-96$ & $U=760.0, z=-2.696, p=0.006^{* *}$ \\
\hline Disease duration & 6.0 & $1-13$ & 3.5 & $0-28$ & $U=869.5, z=-2.039, p=0.041^{*}$ \\
\hline Hoehn and Yahr & 3.0 & $1-5$ & 2.5 & $1-5$ & $U=712.5, z=-1.585, p=0.114$ \\
\hline MMSE & 28 & $10-30$ & 28 & $0-30$ & $U=1115.5, z=-0.502, p=0.619$ \\
\hline UPDRS & 37 & $5-60$ & 32 & $4-89$ & $U=939.0, z=-1.618, p=0.106$ \\
\hline Males & $n=6$ & & $n=53$ & & \\
\hline Grip strength (kg) & 16.0 & $10-29$ & 21.0 & $6-41$ & $U=89.0, z=-1.206, p=0.239$ \\
\hline MAC (cm) & 23.0 & $19-36$ & 29.0 & $22-36$ & $U=72.5, z=-1.543, p=0.129$ \\
\hline TSF $(\mathrm{mm})$ & 7 & $5-11$ & 11 & $4-25$ & $U=61.0, z=-1.920, p=0.055$ \\
\hline MAMC (cm) & 21.0 & $16.5-33.8$ & 25.2 & $18.7-32.2$ & $U=74.0, z=-1.447, p=0.155$ \\
\hline Unintentional weight loss & \multicolumn{2}{|c|}{1 yes, 5 no } & \multicolumn{2}{|c|}{5 yes, 48 no } & $\chi^{2}(1)=0.309, p=1.000$ \\
\hline Females & $n=15$ & & $n=62$ & & \\
\hline Grip strength (kg) & 11.0 & $5-19$ & 15.0 & $0-28$ & $U=301.0, z=-2.074, p=0.040^{*}$ \\
\hline MAC (cm) & 22.0 & $17-26$ & 28.0 & $16-38$ & $U=94.0, z=-4.670, p<0.001^{* *}$ \\
\hline TSF (mm) & 10 & $8.0-14.0$ & 14 & $6-37$ & $U=221.5, z=-3.044, p=0.002^{* *}$ \\
\hline MAMC $(\mathrm{cm})$ & 18.7 & $14.3-23.3$ & 22.2 & $12.2-33.8$ & $U=158.5, z=-3.775, p<0.001^{* *}$ \\
\hline Unintentional weight loss & \multicolumn{2}{|c|}{8 yes, 7 no } & \multicolumn{2}{|c|}{6 yes, 56 no } & $\chi^{2}(1)=15.474, p=0.001^{* *}$ \\
\hline
\end{tabular}

* Significant at the $p<0.05$ level.

** Significant at the $p<0.01$ level.

affected by the disease. Once again, BMI and TSF were lower and MAC higher than for our cohort. Our study of patients at an apparently earlier disease stage tends to support the view that those earlier in the disease cycle have fewer problems of under-nutrition, as noted by others [12]. Further research in this area is required.

Measurements of grip strength, MAC and TSF were highly correlated with having a BMI below 20 in females, but not males. Consideration of median and range of the data suggests that this lack of statistical significance is due to the relatively low number of males with BMI below 20, rather than any inherent anthropomorphic reason. Anthropomorphic measurements may offer an alternative method of assessing PD patients for nutritional risk, where height and weight are difficult to assess. Indeed, anthropomorphic measurements have been shown to be significant predictors of mortality in older people [28].

Whilst these are prevalence studies, we accept that the prevalence estimate is only a minimum estimate and that not all cases of PD will necessarily have been identified, and that not all of the prevalent population

Table 3 Comparison of our cohort with reference data from other studies

\begin{tabular}{|c|c|c|c|}
\hline & $\begin{array}{l}\text { North Tyneside and North } \\
\text { Northumberland PD patients (median) }\end{array}$ & Edinburgh $75-79$ years (mean) ${ }^{23}$ & South Wales $75-79$ years (median) ${ }^{24}$ \\
\hline \multicolumn{4}{|l|}{ Males } \\
\hline BMI $\left(\mathrm{kg} / \mathrm{m}^{2}\right)$ & 24.8 & 26.9 & 23.9 \\
\hline MAC (cm) & 29.0 & 29.7 & 24.9 \\
\hline TSF $(m m)$ & 10.5 & 11.4 & 7.0 \\
\hline MAMC (cm) & 24.8 & 26.1 & 22.1 \\
\hline \multicolumn{4}{|l|}{ Females } \\
\hline BMI $\left(\mathrm{kg} / \mathrm{m}^{2}\right)$ & 25.0 & 26.2 & 26.1 \\
\hline MAC (cm) & 27.0 & 29.9 & 24.9 \\
\hline TSF $(\mathrm{mm})$ & 13.0 & 17.1 & 14.6 \\
\hline $\operatorname{MAMC}(\mathrm{cm})$ & 21.8 & 24.5 & 20.0 \\
\hline
\end{tabular}


participated in the nutritional study. We suspect the real prevalence of under-nutrition to be even higher taking into account that those with cognitive impairment and severe disability were more likely to be excluded.

\section{Conclusions}

Under-nutrition is a problem for $15 \%$ of a prevalent population of community-dwelling people with PD in North-East England and almost a quarter $(23.5 \%)$ are at medium or high risk of malnutrition. Women are at greater risk from severe under-nutrition than men. However, the prevalence of under-nutrition increases with age and disease duration. Clinicians should screen for, and monitor, nutrition in all PD patients and should consider use of the MUST screening tool as an early indicator of nutritional problems.

\author{
Acknowledgements \\ This work was supported by Northumbria Healthcare NHS Trust who funded \\ Dr Bob Porter to carry out the North Tyneside prevalence study. Elizabeth J. \\ Turnbull carried out this work in part fulfillment of a medical degree at \\ Newcastle University. We would like to thank the Northumbria PD team, for \\ their help with the project, and also the patients and carers who took part \\ in the project.
}

\section{Author details}

'Department of Medicine, The Royal Victoria Infirmary, Newcastle upon Tyne, NE1 4LP, UK. '²epartment of Medicine, North Tyneside General Hospital, Rake Lane, North Shields, Tyne and Wear, NE29 8NH, UK. ${ }^{3}$ The Medical School, University of Newcastle-upon-Tyne, NE1 7RU, UK.

\section{Authors' contributions}

This study was conceived, organised and executed by Prof. Richard Walker and Dr Bob Porter. Primary data collection was by Dr Bob Porter for North Tyneside and by Prof. Richard Walker and research nurses Catherine Jones and Sheila Durkin for North Northumberland. Statistical analysis and writing of the first draft of the paper was done by Dr William Gray, Dr Ahmed Jaafar and Miss Elizabeth J. Turnbull. All listed authors were involved in the preparation, review and critique of the final manuscript.

\section{Competing interests}

The authors declare that they have no competing interests.

Received: 10 September 2010 Accepted: 30 December 2010 Published: 30 December 2010

\section{References}

1. Beyer PL, Palarino MY, Michalek D, Busenbark K, Koller WC: Weight change and body composition in patients with Parkinson's disease. J Am Diet Assoc 1995, 95:979-983.

2. Barichella M, Cereda E, Pezzoli G: Major nutritional issues in the management of Parkinson's disease. Mov Disord 2009, 24:1881-1892.

3. Levi S, Cox M, Lugon M, Hodkinson M, Tomkins A: Increased energy expenditure in Parkinson's disease. Br Med J 1990, 301:1256-1257.

4. Wang $G$, Wan $Y$, Cheng $Q$, et al: Malnutrition and associated factors in Chinese patients with Parkinson's disease: Results from a pilot investigation. Parkinsonism Relat Disord 2010, 16:119-123.

5. Davies KN, King D, Davies H: A study of the nutritional status of elderly patients with Parkinson's disease. Age Ageing 1994, 23:142-145.

6. Durrieu G, Llau M, Rascol O, Senard JM, Rascol A, Montastruc JL: Parkinson's disease and weight loss: a study with anthropometric and nutritional assessment. Clin Auton Res 1992, 2:153-157.

7. Nozaki S, Saito T, Matsumura T, Miyai I, Kang J: Relationship between weight loss and dysphagia in patients with Parkinson's disease. Rhinsho Shinkeigaku 1999, 39:1010-1014.
8. Waxman MJ, Durfee D, Moore M, Manratz RA, Koller W: Nutritional aspects and swallowing function of patients with parkinsons's disease. Nutr Clin Pract 1990, 5:196-199.

9. Andersson I, Sidenvall B: Case studies of food shopping, cooking and eating habits in older women with Parkinson's disease. J Adv Nurs 2001, 35:69-78.

10. Hotta M, Nemoto S, Nomura Y, Suzuki T, Kamo T: Changes in digestive tract function in Parkinson's disease patients receiving antiparkinson agents. Mov Disord 2002, 17(Suppl 5):S141.

11. Wade DN, Mearrick PT, Morris JL: Active-Transport of L-Dopa in Intestine. Nature 1973, 242:463-465.

12. Barichella M, Villa MC, Massarotto A, et al: Mini Nutritional Assessment in patients with Parkinson's disease: correlation between worsening of the malnutrition and increasing number of disease-years. Nutr Neurosci 2008, 11:128-134.

13. Porter R, Macfarlane R, Unwin N, Walker R: The prevalence of Parkinson's disease in an area of North Tyneside in the North-East of England. Neuroepidemiology 2006, 26:156-161.

14. Walker RW, Hand A, Jones C, Wood BH, Gray WK: The prevalence of Parkinson's disease in a rural area of North-East England. Parkinsonism Relat Disord 2010, 16:572-575.

15. Hughes AJ, Daniel SE, Kilford L, Lees AJ: Accuracy of clinical diagnosis of idiopathic Parkinson's disease - a clinicopathological study of 100 cases. J Neurol Neurosurg Psychiatry 1992, 55:181-184.

16. Hoehn M, Yahr M: Parkinsonism: Onset, progression and mortality. Neurology 1967, 17:427-442.

17. Fahn S, Elton RL: UPDRS development committee, Unified Parkinson's Disease Rating Scale. In Recent Developments in Parkinson's Disease. Edited by: Fahn S, Marsden CD, Calne DB, Goldstein M. New Jersey: Macmillan; 1987:153-163.

18. Folstein MF, Folstein SE, Mchugh PR: Mini-Mental State - A practical method for grading cognitive state of patients for the clinician. Journal of Psychiatric Research 1975, 12:189-198.

19. Sergi G, Perissinotto E, Pisent $C$, et al: An adequate threshold for body mass index to detect underweight condition in elderly persons: The Italian Longitudinal Study on Aging (ILSA). Journals of Gerontology Series A: Biological Sciences and Medical Sciences 2005, 60:866-871.

20. World Health Organization Expert Committee: Physical status: The use and interpretation of anthropometry Geneva: WHO; 1995.

21. Jelliffe DB: The assessment of nutritional status of the community Geneva: World Health Organisation; 1966.

22. Elia M: Screening for malnutrition: A multidisciplinary responsibility. development and use of the Malnutrition Universal Screening Tool ('MUST') for adults Redditch, UK: Malnutrition Advisory Group (MAG), a Standing Committee of BAPEN; 2003.

23. Burr ML, Phillips KM: Anthropometric Norms in the Elderly. Br J Nutr 1984, 51:165-169

24. Bannerman E, Reilly JJ, MacLennan WJ, Kirk T, Pender F: Evaluation of validity of British anthropometric reference data for assessing nutritional state of elderly people in Edinburgh: cross sectional study. Br Med J 1997, 315:338-341.

25. Cook Z, Kirk S, Lawrenson S, Sandford S: Use of BMI in the assessment of undernutrition in older subjects: reflecting on practice. Proc Nutr SoC 2005, 64:313-317.

26. Perissinotto E, Pisent C, Sergi G, Grigoletto F, Enzi G: ILSA Working Group. Anthropometric measurements in the elderly: age and gender differences. Br J Nutr 2002, 87:177-186.

27. Markus HS, Tomkins AM, Stern GM: Increased prevalence of undernutrition in Parkinson's disease and its relationship to clinical disease parameters. J Neural Transmission 1993, 5:117-125.

28. Phillips P: Grip strength, mental performance and nutritional status as indicators of mortality risk among female geriatric patients. Age Ageing 1986, 15:53-56.

\section{Pre-publication history}

The pre-publication history for this paper can be accessed here: http://www.biomedcentral.com/1471-2377/10/124/prepub

doi:10.1186/1471-2377-10-124

Cite this article as: Jaafar et al:: A cross-sectional study of the nutritional status of community-dwelling people with idiopathic Parkinson's disease. BMC Neurology 2010 10:124. 